Group Stadistic

\begin{tabular}{|ll|r|r|r|r|r|r|}
\hline & Group & N & Mean & $\begin{array}{r}\text { Standar } \\
\text { deviation }\end{array}$ & $\begin{array}{c}\text { Mean } \\
\text { Standar } \\
\text { error }\end{array}$ & $\begin{array}{r}\text { U Mann } \\
\text { Whitney }\end{array}$ & $\begin{array}{r}\text { T Student } \\
\text { Levene0,3 }\end{array}$ \\
\hline Age now & control & 12 & 13,5342 & 1,80952 &, 52236 & & \\
Evolution & intervention & 12 & 14,7092 & 1,24086 &, 35821 & & \\
time & intervention & 12 & 6,1833 & 3,79014 & 1,09412 & & \\
HbA1c before & control & 12 & 8,2333 & 4,47424 & 1,29160 & & \\
Accucheck & intervention & 12 & 8,0750 &, 30488 &, 08801 & & \\
\hline dif_0_3m & control & 12 &,- 0667 &, 68799 &, 19861 &, 799 &, 685 \\
& intervention & 12 &,- 1667 &, 48492 &, 13999 & & $95 \%$ IC $0,40-0,60$ \\
dif_3_6m & control & 12 &,- 0917 &, 84473 &, 24385 &, 291 &, 502 \\
& interventionn & 12 &,- 3000 &, 63389 &, 18299 & & $95 \%$ IC $0,42-0,84$ \\
dif_0_6m & control & 12 &,- 1583 &, 36794 &, 10621 &, 266 &, 196 \\
& intervention & 12 &,- 4667 &, 71138 &, 20536 & &, $85 \%$ IC $0,18-0,79$ \\
\hline
\end{tabular}

\section{PO-0064 CYSTIC FIBROSIS RELATED DIABETES IN ADOLESCENTS}

${ }^{1}$ D Virlan, ${ }^{1} \mathrm{~L}$ Sochirca, ${ }^{2} \mathrm{~A}$ Chiriac, ${ }^{3} \mathrm{~S}$ Sciuca. ${ }^{1}$ Department of Pediatrics, State Medical and Pharmaceutical University Nicolae Testemitanu, Chisinau, Moldova; ${ }^{2}$ Department of Endocrinology, National Children's Hospital "Emilian Cotaga", Chisinau, Moldova; ${ }^{3}$ Cystic Fibrosis Center, Mother and Child Center, Chisinau, Moldova

\subsection{6/archdischild-2014-307384.736}

Background Cystic fibrosis related diabetes (CFRD) is a combination between reduced insulin secretion and peripheral insulin resistance that only people with cystic fibrosis can get. CFRD is associated with a decline in lung function, poor nutritional status and high mortality rate.

Aim Study of the clinical course and therapeutic management in adolescents with CFRD.

Methods We present 2 cases of CFRD in a female patient (15 years old) and a male patient (17 years old) hospitalised at the CF Centre. The diagnosis of CF was confirmed by positive sweat test (Macroduct USA), identification of CFTR mutation (F508del/F508del), small amounts of elastase in stool. Confirmation of diabetes was achieved by a blood glucose test, blood glucose profile, glycosylated $\mathrm{Hb}, \mathrm{C}$ peptide, glucose and ketones in urine.

Results On the background of pulmonary exacerbation, both patients had hyperglycemia $(9.8 / 14.1 \mathrm{mmol} / \mathrm{l})$. Subsequently, it triggered clinical syndromes suggestive for diabetes - polydipsia, polyuria, weight loss. Glycemic profile variations 7.8-15.8 $\mathrm{mmol} / \mathrm{l}$ in boy and $10.4-21.0 \mathrm{mmol} / \mathrm{l}$ in girl. Glycosylated $\mathrm{Hb}$ values were high $(7.3 / 14.1 \%)$ and C-peptide values were low $(0.624 / 0.513)$. Glucose concentration in urine was 7.3/37.1 g/l and ketones was not detected. Diabetes treatment was performed with Insulin (Glargine, Aspart, Human), which produced clinical benefits by achieving glycemic and clinical syndromes control. Conclusions Patients with CFRD shows a specific clinical framework and require a strict medical diet control and surveillance of the insulin therapy, case that differs from the other types of diabetes. If early introduced, it significantly improves life expectancy towards these patients.

\section{PO-0065 VITAMIN D STATUS IN CHILDREN WITH TYPE 1 DIABETES}

N Makieieva, V Tsymbal, T Ishchenko, N Buzhynska. Pediatrics \#1 and Neonatology, Kharkov National Medical University, Kharkov, Ukraine

\subsection{6/archdischild-2014-307384.737}

Background and aims Serum vitamin D (sVD) deficiency may contribute to the development and progression of diabetic nephropathy (DN). Research has shown urinary vitamin D binding protein ( $\mathrm{UVDBP})$ excretion is increased after renal injury, and is associated with tubulointerstitial damage. Animal studies suggested that SVD deficiency may be associated with urine loss due to kidney damage. The aim was to test associations of sVD with levels of uVDBP, urinary vitamin D (uVD).

Methods 42 children aged 6-17 years with type 1 diabetes were examined: 24 nornoalbuminuric patients (1st group) and 18 microalbuminuric (2nd group). 15 healthy children were included in controls. We measured serum and urine $25(\mathrm{OH}) \mathrm{D}$ levels, uVDBP concentrations and tested their correlations.

Results sVD levels were decreased in the patients of the 1st and 2nd groups, compared with controls $((22.03(17.23 ; 24.44)$ and 14.42 (12.02; 19.63), compared with 30.65 (28.45; 35.05) ng/ $\mathrm{ml}$, respectively) $(\mathrm{p}<0.001))$. uVDBP levels were elevated in the patients of the 1st and 2nd groups, compared with controls ((179.5 (174.0; 189.0) and $219.0(216.0 ; 222.0)$, compared with $125.0(116.5 ; 136.0) \mathrm{ng} / \mathrm{mg}$, respectively) (p < 0.001)). uVD levels were increased in the patients of the 1st and 2nd groups, compared with control group $((3.2(2.9 ; 3.3)$ and $3.9(3.7 ; 4.1)$, 